\title{
Case Report: Investigating Possible Etiologies of Post- (4) Exposure Prophylaxis Failure and Deaths From Rabies Infection: Case Reports
}

\author{
Azadeh Rasooli' ${ }^{1}$ Behzad Pourhossein ${ }^{2,3}$, Rouzbeh Bashar ${ }^{3}$, Mohammad Reza Shirzadi $^{4}$, Behzad Amiri ${ }^{4}$, Elmira Vadaye Kheiri ${ }^{3}$, Babak \\ Mostafazadeh ${ }^{5,6}$, Maryam Fazeli ${ }^{3 *}$ (i)
}

1. Department of Biochemistry, Faculty of Sciences, Payame-Noor University, Tehran, Iran.

2. Department of Virology, School of Public Health, Tehran University of Medical Sciences, Tehran, Iran.

3. Department of Virology, Pasteur Institute of Iran, Tehran, Iran.

4. Department of Zoonosis Control, Center for Communicable Diseases Control, Ministry of Health and Medical Education, Tehran, Iran.

5. Department of Forensic Medicine and Toxicology, Loghman Hakim Hospital, Shahid Beheshti University of Medical Sciences, Tehran, Iran.

6. Toxicological Research Center, Shahid Beheshti University of Medical Sciences, Tehran, Iran.

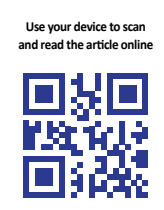

Citation: Rasooli A, Pourhossein B, Bashar R, Shirzadi MR, Amiri B, Vadaye Kheiri E, Mostafazadeh B, Fazeli M. Investigating Possible Etiologies of Post-Exposure Prophylaxis Failure and Deaths From Rabies Infection: Case Reports International Journal of Medical Toxicology and Forensic Medicine. 2020; 10(3):27378. https://doi.org/10.32598/ijmtfm.v10i3.27378

doi) $h$ https://doi.org/10.32598/ijmtfm.v10i3.27378

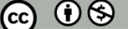

Article info:

Received: 07 Oct 2019

First Revision: 17 Oct 2019

Accepted: 12 Nov 2019

Published: 28 Sep 2020

\section{Keywords:}

Rabies, Rabies vaccine Post-exposure prophylaxis, Lyssavirus

\section{ABSTRACT}

Rabies virus, as a neurotropic agent, is transmitted to humans usually after animal biting. Rabies is endemic in most Middle Eastern countries. Pre-exposure Prophylaxis (PrEP) program is recommended for high-risk populations in endemic areas. The present study reported 7 deaths from rabies infection despite human Post-Exposure Prophylaxis (PEP) and the evolution of their possible etiologies from 2014 to 2018 in Iran. In this study, 29 rabid human deaths were evaluated despite PEP in 2014-2018. Seven people deceased despite receiving PEP. The most damaged organs were hands and face (71.43\%). Injecting anti-Rabies Immunoglobulin (RIG) around the wound, improper cleansing, and delayed PEP were the main causes of PEP failure. In addition, immunodeficiency in a patient was another cause of failure. Our obtained data suggested that immediate precise measures after exposure based on the World Health Organization (WHO) recommendation, maintaining the temperature integrity (cold chain) of vaccines, and RIGs during transportation, and performing detailed injection schedule could prevent PEP failure in most cases. Furthermore, society's awareness plays a key role in controlling the disease, especially in endemic areas.

* Corresponding Author:

Maryam Fazeli, PhD.

Address: Department of Virology, Pasteur Institute of Iran, Tehran, Iran.

Tel: +98 (21) 66403496

E-mail: m fazeli@pasteurac.ir 


\section{Introduction}

abies virus is a neurotropic virus with a single-stranded a single-stranded $\mathbf{R}$ RNA belonging to the Lyssavirus genus and the Rhabdoviridae family [1]. It is estimated that rabies disease annually causes 3-6×104 deaths due to fatal encephalomyelitis, especially in Asian and African countries [2]. This virus is mainly transmitted to humans following a bite by a rabid animal [3]. The incubation period of the disease in humans usually ranges from a few days to several months. Changes in this period are related to different factors, including the distance of injured site to the central nervous system, the number of inoculated viruses, the severity of injuries, virus virulence, as well as the age and immune system status of the infected person [3].

A combination of vaccine and anti-Rabies Immunoglobulin (RIG), based on the World Health Organization (WHO) protocol, could prevent post-exposure human rabies. However, after the onset of clinical symptoms, rabies treatment is usually impossible and the prognosis of the disease is negative [4]. Post-exposure Prophylaxis (PEP) would be significantly effective when used immediately and appropriately after biting and following the WHO recommendations.

Vaccines used in Iran based on the approval of the Iranian national committee of control and surveillance of rabies is Vero Rabies Vaccine (PVRV); it is inactivated and obtained from the culture of WISTAR PM/WI 3815083-3M rabies in PVRV cells [5]. This vaccine is distributed under the brand name Rabipur ${ }^{\circledR}$ and is currently the most commonly used vaccine worldwide [5].

PEP for rabies is provided free of charge in Iran and 700 centers for rabies PEP are actively working 24/7; therefore, there are limited human mortalities reported annually among high rates of animal rabies. In Iran, rabies is enzootic in a dog as well as in wildlife. Annually, $35-45 \times 104$ doses of inactivated rabies vaccine are used to immunize domestic animals [6]. Detecting human mortality reports, even after the control and prevention measures, was the main purpose of this study. We aimed to investigate the possible etiology of rare cases of human PEP failure among individuals bitten by rabid animals in different regions of Iran in 2014-2018.

\section{Case report}

This study investigated all suspected or confirmed cases of rabies despite PEP, based on the protocol provided by the Iranian Center for Disease Control (CDC) [7] from January 2014 to December 2018. In total, 29 deaths due to rabies were recorded in the study period. Seven individuals had received a vaccine and RIG. All deceased cases were male and the youngest and oldest ones aged 10 and 67 years, respectively. The most damaged organs were hands and face $(71.43 \%)$. The demographic and PEP treatment data of deceased ones with non-compliance items with WHO guidelines are listed in Table 1. The vaccine licensed for rabies PEP in Iran is Verorab $₫$ (Sanofi Pasteur Ltd.), i.e., approved by the WHO (potency $\geq 2.5$ ). The possible causes of death are evaluated in detail in the discussion section.

For antemortem diagnosis, the qRT-PCR test was performed on saliva samples and the direct Fluorescence Antibody Test (FAT) was conducted on nuchal skin biopsy. In this study, the FAT test was performed on the brain samples for the postmortem diagnosis of rabies. Besides, in cases of negative results in the FAT test during postmortem diagnosis, Mouse Inoculation Test (MIT) was conducted as a complementary technique on laboratory sensitive animals.

In this study, required data, such as demographic information, patients' medical information, disease history, all received treatment, and vaccines were collected through the patient database, available at the zoonosis department of the CDC.

From a rabies suspected-patient with a history of being bitten with rabid animals or suspected rabies, at least three saliva samples with 0.5 to $1 \mathrm{~mL}$ volume per sample at intervals of 3 to 6 hours were obtained using plastic dropper 4-6 days after the onset of clinical symptoms. Then, all samples were maintained in a well-sealed container without any preservative material transferred to the laboratory with maintaining temperature integrity (cold chain).

A section of skin with a diameter of 5-6 mm containing a minimum of 10 follicles from the posterior region of the neck with the hairline of each rabies suspected patient was collected using a puncture. Preservatives or additional fluids were not added. Prepared biopsies on a small piece of sterilized gauze wetted with a physiological serum were put into a fully-sealed container approved by the WHO. Then, they were provided within 24 hours with maintaining temperature integrity (cold chain). 
+
$\overrightarrow{0}$
0
0
0
0
$=$
0
0
0
0
0
0
0
0
0
0
0
0
0
0
0
0
0
0
0
0
0
0
0
0
0
0
0

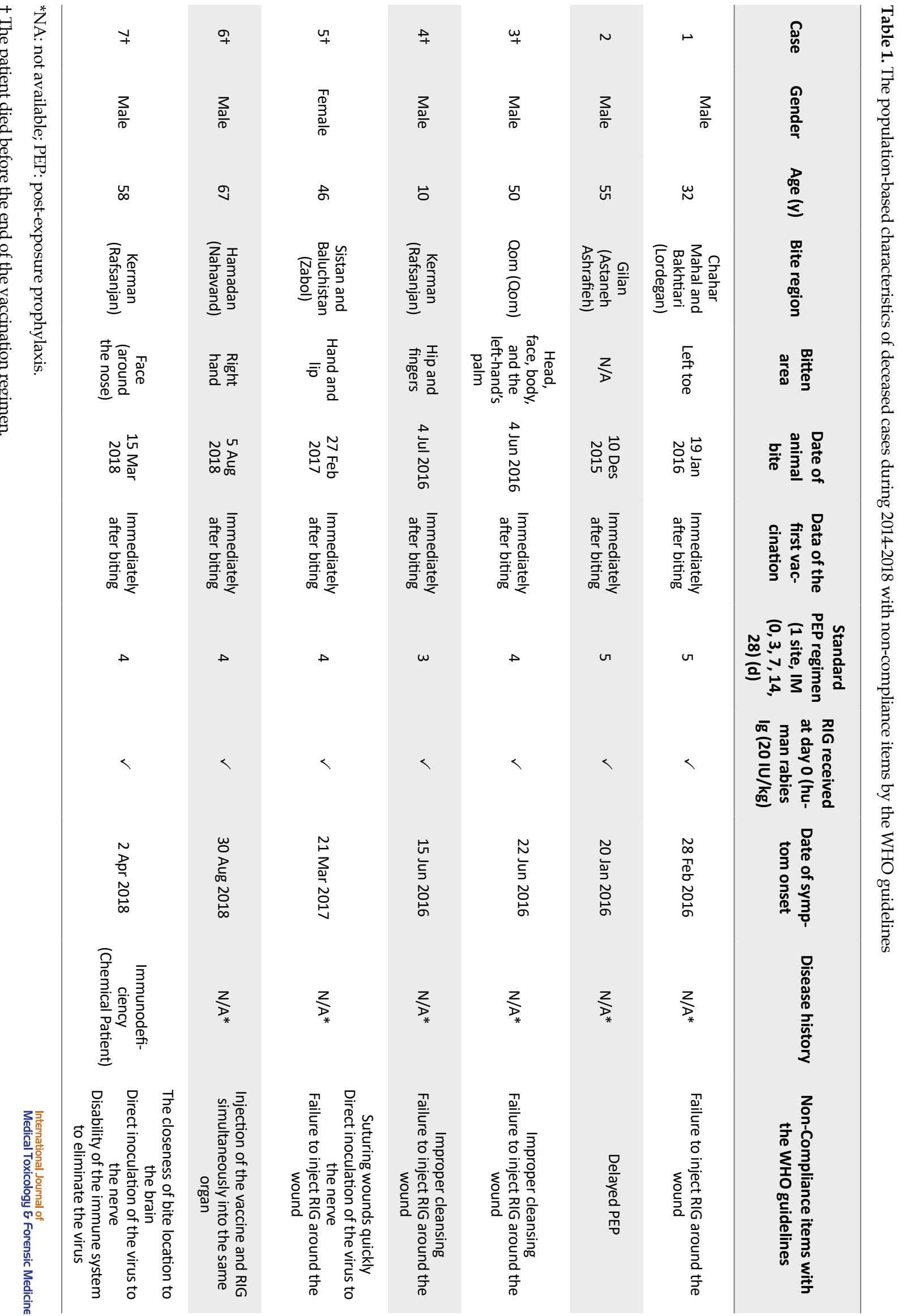




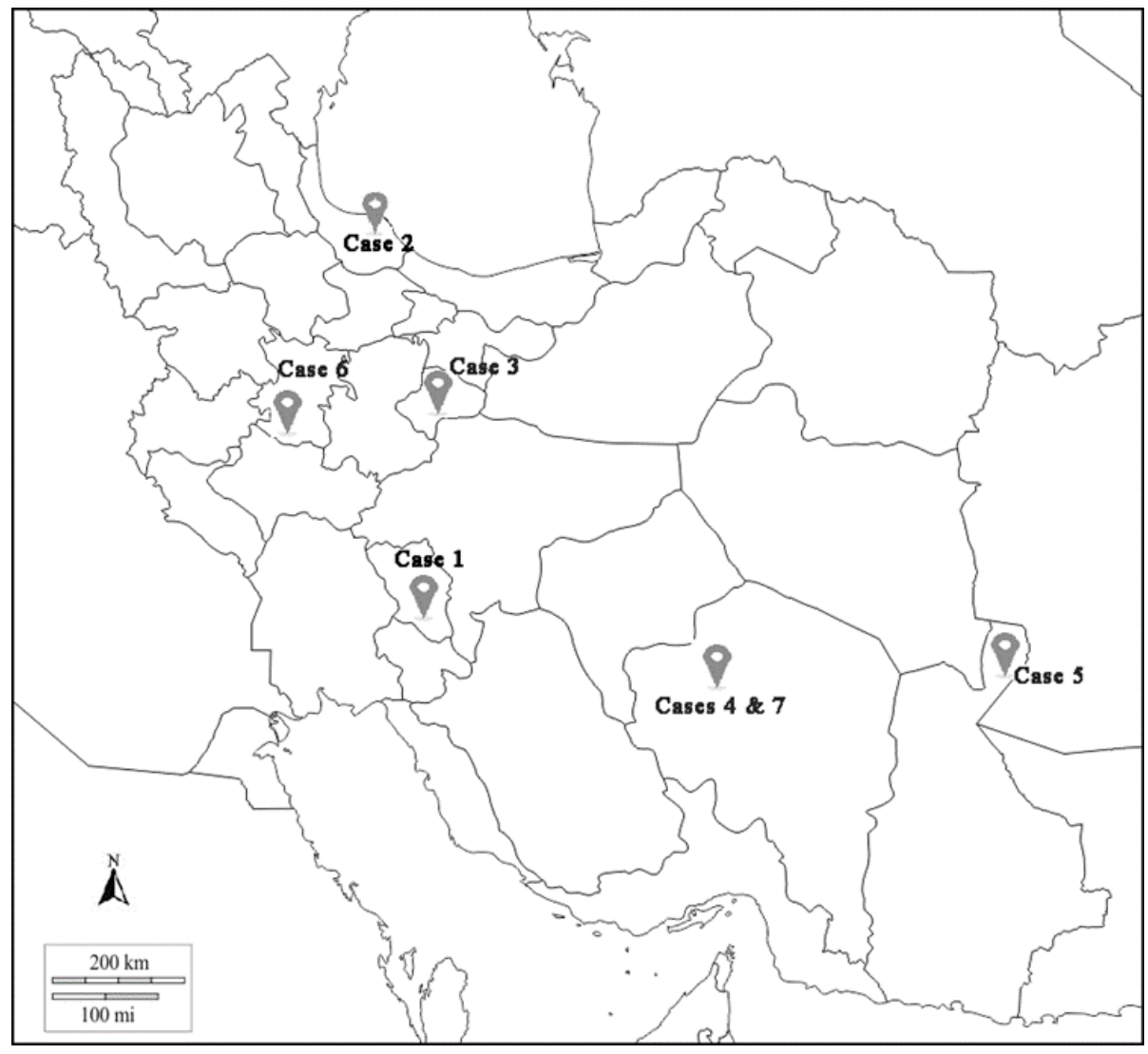

Figure 1. The geographical distribution of deaths due to PEP failure International Journal of
Medical Toxicology \& Forensic Medicine

A small piece of the brain tissue (about two $\mathrm{cm}$ ) was obtained by pushing the plastic straw into foramen magnum (occipital foramen) [8]. Next, it was transported to the national reference laboratory for rabies diagnosis using the WHO-approved method, in a fully-sealed sample container.

Three saliva samples of each patient were used after pooling together for total RNA extraction by Trizol, according to the manufacturer's instructions (Invitrogen, Carlsbad, California). Then, total RNA was quantified at Optical Density (OD) of 260 and $280 \mathrm{~nm}$ by nanodrop 2000 spectrophotometer (Thermo Fisher, USA). A total of 50 to $100 \mathrm{ng}$ viral RNA was first converted to cDNA using reverse transcriptase-polymerase chain reaction. Synthesized RNA was stored at $-80^{\circ} \mathrm{C}$. To synthesize cDNA, Thermo (scientific) Lithuania kit was used based on the available protocol in the kit. The cDNA was stored for the qRT-PCR step at $-20^{\circ} \mathrm{C}$. The qRT-PCR assay was performed using Superscript III Platinum One-Step qRTPCR (Invitrogen) kit based on the instructions in the kit and designed primers [9]. The FAT test was conducted according to the instructions. In the case of a negative result, the MIT test was performed as a complementary technique [10].

\section{Discussion}

Following a suspicious bite, PEP significantly prevents mortality. Besides, it directly accounts for the highest economic burden of rabies control in Iran, per year [11]. The WHO recommends implementing cell culture vaccines. There are some reports related to the failure of this vaccine or its constant serious complications, such as encephalitis, encephalomyelitis, myeloradiculitis, and polyradiculitis within 4-7 months [12].

Death due to rabies occurs when a bitten person by an animal suspected to be rabid fails to receive the necessary PEP. These PEP include cleansing, complete vaccination period, and RIG if necessary. RIG is expensive and not always available; it is often not used despite the strong recommendation, which sometimes leads to PEP failure. Moreover, not injecting RIG in each wound is another cause of PEP failure. Besides, failure to follow the WHO guidelines, such as timetable, delayed PEP, and improper wound care, including hidden small wounds, resulting in a high incidence of rabies-associated mortality [13]. These PEP failures should not be mistakenly considered as vaccine failure. Rarely, PEP failure has occurred despite fulfilling all the recommendations. Its causes in- 
clude host immune responses, pathogenic characteristics (pathogenicity \& genetic variation), the location of virus entry (e.g., face biting near the brain and in rare cases, direct inoculation of the virus to nerves), and viral load. PEP failure has also been observed in the cases of employing the WHO unauthorized-vaccine that have inadequate potential to elicit immunity against rabies. A study was conducted on animal vaccines in Italy. The relevant data indicated that the choice of vaccine had a significant impact on the failure or success of the rabies vaccination [14]. The fast and proper care of all wounds, maintaining temperature integrity along a cold chain during transport of vaccines, RIGs, and to perform detailed injection schedule could prevent PEP failure. The best condition to maintain the vaccine is to store it at low temperatures around $2-8^{\circ} \mathrm{C}$.

Corticosteroids, other immunosuppression drugs, antimalarial medications, and diseases that induce immunosuppression could interfere with active immunization after vaccination. Pre-exposure Prophylaxis (PrEP) should be prescribed for immunocompromised patients, considering that the produced immune response may be inadequate. Additionally, their antibody level should be checked after the prescription of PrEP. Consulting with the $\mathrm{CDC}$ should be considered if the antibody level in a patient after receiving three injections of the vaccine is inadequate.

Neutralizing antibodies are generally produced against rabies virus $\mathrm{G}$ protein. Accordingly, the mutations necessary to escape the virus from the neutralizing antibodies have adverse effects on the protein $\mathrm{G}$ function; thus, they produce defective viruses. Such mutations have rarely been detected in Rhabdovirus. However, some studies have suggested that antigenic changes in $G$ protein among street viruses cause rabies vaccination failure [15]. The following items are some possible causes of PEP failure in the existing case studies.

Case resolutions: the evaluation of PEP failure causes in the study period

Society awareness: Society awareness about rabies and the correct manner of management by individuals after exposure in the pre-clinic stage is the first step to success in the control of the disease. As per Table 1, in the second case, the patient delayed in referring to the center for receiving the vaccine and RIG.

Inappropriate or inadequate cleansing: In this study, in cases 3 and 4 (Figure 1), it seems that several numbers of the reported deaths occurred due to inappropriate or inadequate cleansing even if the vaccine and RIG were injected promptly. Sunlight, drying, detergents (e.g., soap), and other disinfectants inactivate the rabies virus. Wound care is necessary to prevent rabies infection. Each wound should be disinfected with soap and water, or if available, iodine solution, $40 \%-70 \%$ alcohol, $0.1 \%$ cetrimide or similar compounds, or anti-virus agents like Povidone. Besides, if necessary, wounds should be disinfected with a local anesthetic. Animal experiments revealed that initial effective wound cleansing could effectively prevent rabies infections [16]. Moreover, this category could be accounted for social awareness about disease prevention.

As reported in case 6 (Figure 1), the administration of RIG and vaccine in the same organ was among the possible causes of PEP failure in some studied patients. This issue may be especially observed in type III injuries occurring in the hand. This problem could occur due to the presence of primary healthcare staff or even, inadequate in-service training of control and prevention center personnel. According to the WHO recommendation, when one hand is bitten, RIG should be administrated in the bitten organ; a dose of vaccine should also be injected into the deltoid muscle of the opposite arm on day 0 , and the second dose of the vaccine should be injected into the anterior thigh muscle. Otherwise, vaccines should be administrated based on the five-dose 'Essen' regimen. Upon vaccine administration, humoral immunity is activated and causes the production of neutralizing antibodies against rabies. RIG administration could enhance immune response while the level of humoral immunity in the body remains inadequate to prevent rabies infection [4].

The case number 7 (Figure 1) was an Iranian chemical warfare veteran with advanced immunodeficiency; he had been receiving immunosuppressive medicine for a long time. There is a possibility that immune responses against rabies have not increased after the administration of rabies vaccine. Additionally, the intradermal injection should not be used for all patients, especially immunocompromised ones or those who use steroids and antimalarial medications [17].

In the case one (Figure 1), although the bite area was in the left toe and caused a small wound, the presence of symptoms was due to the injection of serum in gluteal muscle rather than injection at the wound site. RIG should never be injected into the gluteal muscles, as it may not induce an appropriate defense against the injected virus [18]. A study revealed that only RIG injection around the wound could prevent death from rabies 
in individuals who have been severely bitten by dogs. This incidence follows a sharp decline in RIG consumption. RIG should be carefully administrated to cover the entire wound surface to reach its depth. Gluteal region is not recommended for the rest of the RIG administration, i.e., calculated according to the WHO guidelines. This is due to the poor absorption of antigen from the fat present in this region and the inability to produce the optimal immune responses [19].

A cause of PEP failure is the direct inoculation of the virus into nerve endings. Thus, a wound in the face with numerous nerves may be a possible cause of prophylaxis failure. This issue could be a potential cause of PEP failure in cases 5 and 7.

A bite wound should never be stitched, except in specific circumstances or cut in the main artery; this is because the rabies virus is highly sensitive and loses its pathogenicity easily after exposure to light and oxygen. In case of bleeding due to biting, RIG should be injected immediately into the site. In this study, case number 5 received five doses of vaccine and RIG; however, death occurred after one month, which could be due to a rapid suture of the wound site. Stitching the wound could increase the speed of the virus spreading and entering to peripheral nerves and cause them to escape from the immune system [20].

\section{Conclusion}

The presentation of cases in this study suggested that the lack of social awareness about the infection and the correct manner of managing it by individuals and the control and prevention center personnel after exposure in the pre-clinic stage was the main cause of PEP failure in controlling the disease. Rabies usually occurs in remote areas of developing or poor countries where individuals usually have inadequate education. The inaccurate management of the vaccine maintenance and the inappropriate use of it by those without appropriate training in this area could always be problematic. Endemic countries should improve awareness level, as well as access to health care, medicines, and vaccines for populations at risk.

\section{Ethical Considerations}

\section{Compliance with ethical guidelines}

This article does not contain any interventions on human or animal by any of the authors. In addition, infor- mation about the human case study group is anonymous in this study.

\section{Funding}

This research did not receive any grant from funding agencies in the public, commercial, or non-profit sectors.

\section{Authors' contributions}

All authors were equally contributed in preparing this article.

\section{Conflict of interest}

The authors declared no conflicts of interest.

\section{Acknowledgements}

We would like to thank all colleagues in the National collaborating center for reference and research on rabies, Pasteur Institute of Iran involved in this study, Zoonoses Control Department, Center for Communicable Diseases Control, Ministry of Health and Medical Education, Tehran, Iran for their supports.

\section{References}

[1] Knobel DL, Cleaveland S, Coleman PG, Fèvre EM, Meltzer MI, Miranda MEG, et al. Re-evaluating the burden of rabies in Africa and Asia. Bulletin of the World health Organization. 2005; 83:360-8.

[2] Schnell MJ, McGettigan JP, Wirblich C, Papaneri A. The cell biology of rabies virus: Using stealth to reach the brain. Nat Rev Microbiol. 2010; 8(1):51-61. [DOI:10.1038/nrmicro2260] [PMID]

[3] Sterner RT, Smith GC. Modelling wildlife rabies: Transmission, economics, and conservation. Biol conserv. 2006; 131(2):163-79. [DOI:10.1016/j.biocon.2006.05.004]

[4] WHO. WHO expert consultation on rabies: Third report.World Health Organization; 2018.

[5] Wu X, Smith TG, Rupprecht CE. From brain passage to cell adaptation: The road of human rabies vaccine development. Expert Rev Vaccines. 2011; 10(11):1597-608. [DOI:10.1586/ erv.11.140] [PMID]

[6] Janani A, Fayaz A, Simani S, Farahtaj F, Eslami N, Howaizi $\mathrm{N}$, et al. Epidemiology and control of rabies in Iran. Dev Biologicals. 2008; 131:207-11.

[7] Golahdooz M, Eybpoosh S, Bashar R, Taherizadeh M, Pourhossein B, Shirzadi M, et al. Comparison of immune responses following intradermal and intramuscular rabies vaccination methods. JoMMID. 2018; 6(4):77-86. [DOI:10.29252/ JoMMID.6.4.77] 
[8] Tong TR, Leung KM, Lee K, Lam AW. Trucut needle biopsy through superior orbital fissure for diagnosis of rabies. Lancet. 1999; 354(9196):2137-8. [DOI:10.1016/S0140-6736(99)95093-6]

[9] Amiri F, Fadajan Z, Rasooli A, Salahshourifar I, Bashar R, et al. Development and Validation of heminested RT-PCR and qRT-PCR assays for comprehensive detection of rabies virus in the suspected rabid brain and saliva samples. Arch Clin Infect Dis. Online ahead of Print. 2019; 14(2):e85790. [DOI:10.5812/archcid.85790]

[10] Meslin F-X, Kaplan MM, Koprowski H, Organization WH. Laboratory techniques in rabies: Geneva: World Health Organization; 1996.

[11] Gholami A, Fayaz A, Farahtaj F. Rabies in Iran: Past, present and future. JoMMID. 2014; 2(1):1-10.

[12] Ahasan HN, Chowdhury MJ, Azhar M, Rafiqueuddin A. Neuroparalytic complications after anti-rabies vaccine (inactivated nervous tissue vaccine). Trop Doct. 1995; 25(2):94. [D OI:10.1177/004947559502500220] [PMID]

[13] Shill M, Baynes RD, Miller SD. Fatal rabies encephalitis despite appropriate post-exposure prophylaxis. N Engl J Med. 1987; 316(20):1257-8. [DOI:10.1056/NEJM198705143162006] [PMID]

[14] Rota Nodari E, Alonso S, Mancin M, De Nardi M, Hudson-Cooke S, Veggiato C, et al. Rabies vaccination: Higher failure rates in imported dogs than in those vaccinated in Italy. Zoonoses Public Health. 2017; 64(2):146-55. [DOI:10.1111/ zph.12268] [PMID]

[15] Wiktor TJ, Koprowski H. Antigenic variants of rabies virus. The Journal of Experimental Medicine. 1980; 152(1):99-112. [DOI:10.1084/jem.152.1.99] [PMID] [PMCID]

[16] Dean D, Baer G, Thompson W. Studies on the local treatment of rabies-infected wounds. Bulletin of the World Health Organization. 1963; 28(4):477.

[17] Gongal G, Sampath G. Introduction of intradermal rabies vaccination-A paradigm shift in improving post-exposure prophylaxis in Asia. Vaccine. 2019; 37(Suppl 1):A94-A98. [DOI:10.1016/j.vaccine.2018.08.034] [PMID]

[18] Hughes G, Smith J, Hanlon C, Rupprecht C. Evaluation of a TaqMan PCR assay to detect rabies virus RNA: Influence of sequence variation and application to quantification of viral loads. J Clin Microbiol. 2004; 42(1):299-306. [DOI:10.1128/ JCM.42.1.299-306.2004] [PMID] [PMCID]

[19] Bharti OK, Madhusudana SN, Wilde H. Injecting Rabies Immunoglobulin (RIG) into wounds only: A significant saving of lives and costly RIG. Hum Vaccin Immunother. 2017; 13(4):762-5. [DOI:10.1080/21645515.2016.1255834] [PMID] [PMCID]

[20] Tinsa F, Borgi A, Jahouat I, Boussetta K. Rabies encephalitis in a child: a failure of rabies post exposure prophylaxis? BMJ case reports. 2015; 2015:bcr2014206191. [DOI:10.1136/bcr2014-206191] [PMID] [PMCID] 